\title{
Nucleation and superstabilization in small systems
}

\author{
T. Philippe* \\ Physique de la Matière Condensée, Ecole Polytechnique, CNRS, Université Paris-Saclay, 91128 Palaiseau, France
}

(Received 5 July 2017; published 21 September 2017)

\begin{abstract}
Phase transitions are known to present peculiarities in small systems that are related to depletion effects of the ambient phase. Mass conservation affects the conditions of thermodynamic equilibrium between a nucleus of the new phase and the matrix as compared with nucleation in infinite systems. This finite-size effect is known to delay the phase transition but can also impede nucleation in very small systems as it stabilizes the initial state, originally metastable in infinite systems. In this work, we investigate this superstabilization effect in the context of classical nucleation theory in multicomponent solutions and we derive an analytical expression for the system size below which nucleation becomes thermodynamically impossible. Comparing with the exact solution, our simple result is shown to accurately predict the superstabilization effect, and can therefore be used, for instance, as a guideline for the design of novel nanomaterials.
\end{abstract}

DOI: 10.1103/PhysRevE.96.032802

\section{INTRODUCTION}

Nanoscience demands a reduction in the size of the systems and in many cases the design of new materials involves a phase transition. The initial and crucial step of a typical phase transition is often the formation of a nucleus from a metastable bulk phase. Thus, fabrication of novel nanomaterials requires an accurate control over the formation of the embryo or nucleus of the new phase. In this case, the fundamental mechanism of the phase transformation is nucleation, which is known to play a very important role in many different fields [1-18]. Small systems that are of scientific and technological interests involve, for instance, nucleation of metallic or semiconductor clusters that can form quantum dots [19-23], or crystallization in small liquid droplets, as for nanowires grown by the vaporliquid-solid mechanism [24], to mention just a few examples.

Phase transformations in confined space can significantly differ from bulk scenarios as depletion effects affect both the thermodynamics and kinetics of first order phase transitions [25-40]. For instance, in a closed cavity the phase diagram of a binary mixture is modified [25]. As small systems receive now increasing attention, many recent works have been dedicated to the understanding of nucleation in a confined space in various theoretical frameworks and contexts. The problem of nucleation in confined systems has been studied in molecular dynamics and Monte Carlo [26], in density functional theory [27], solving the kinetic equations [28-30], or in the context of both classical [31] and generalized Gibbs' nucleation theories $[32,33]$. In the present work, we consider the effect of depletion in small systems that contain only one nucleus (as always in nucleation) but this effect is also of major importance in large systems if the entire population of clusters is considered, and these two aspects are closely related [33]. The main peculiarity in small systems is the possibility of stabilizing the metastable initial macroscopic phase, as a new means to modify nanomaterial properties. Both the size of the critical cluster and the nucleation barrier increase in a confined space as compared with nucleation in an infinite system, thus the phase transition is delayed but as the size

\footnotetext{
*thomas.philippe@ polytechnique.edu
}

of the system decreases nucleation is impeded and the initial state becomes stable [33-35,39]. The finite available mass prohibits nucleation when the system is too small and this effect is known as superstabilization, but generally imposes a maximal supercritical cluster size; this new extremum in the thermodynamic potential associated with the formation of the nucleus corresponds to a stable and final state, as growth is stopped.

In this paper, we investigate the superstabilization effect in multicomponent systems using the capillary model in the context of classical nucleation; in other words we use the macroscopic classical picture underlying the classical nucleation theory and resort to a general thermodynamic description. As mentioned earlier there are many papers that deal with the problem of nucleation in small systems and certainly better theoretical approaches have been employed to determine the critical parameters [25,29,34-37] but they require a numerical method. Our approach is different as our goal is to derive an analytical and simple expression for the system size below which nucleation is thermodynamically prohibited, which can help in the design of nanomaterials. Consequently we work with classical nucleation in the context of macroscopic thermodynamics. The analysis is valid for nondilute and nonideal solutions but is restricted to low supersaturations. We find that our simple result compares well with the exact solution and shows that the knowledge of only a few thermodynamic parameters allows for an accurate prediction of the superstabilization effect. Below we present the main results of our analysis.

\section{NUCLEATION IN THE NPT ENSEMBLE}

Following the Wilhelmsen and Reguera treatment [37], we consider nucleation in the NPT ensemble, where $N$ is the total number of atoms, $P$ is the pressure, and $T$ is the temperature. The system consists of a spherical nucleus with radius $R_{\beta}$ ( $\beta$ phase) in either an infinite matrix $\alpha$ or a spherical domain of the $\alpha$ phase with radius $R_{\alpha}$ (see Fig. 1). The present analysis is restricted to homogeneous nucleation. In the capillary approach, the $\alpha$ and $\beta$ phases are separated by a sharp interface with surface tension $\sigma$. 


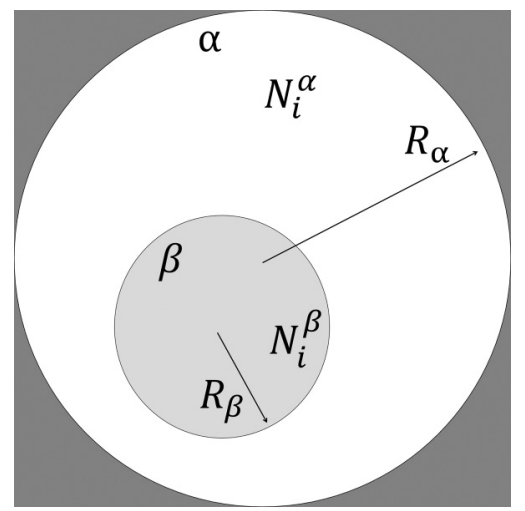

FIG. 1. The confined system consists of a spherical nucleus with radius $R_{\beta}$ ( $\beta$ phase) in a spherical domain of the $\alpha$ phase with radius $R_{\alpha}$.

Due to matter conservation, an important sum rule on the mole (or atomic) fraction of each species exists:

$$
\sum_{i=1}^{k} c_{i}^{j}=1 \quad j=\alpha, \beta,
$$

where $k$ is the total number of components. $c_{i}^{j}=N_{i}^{j} / N^{j}$ is the mole fraction of species $i$ in the $j$ phase. $N_{i}^{j}$ and $N^{j}$ are respectively the number of atoms $i$ and the total number of atoms in the $j$ phase. The total number of atoms of species $i$ in the system $\left(N_{i}\right)$ is equal to $N_{i}^{\alpha}+N_{i}^{\beta}$.

In the $N P T$ ensemble the proper thermodynamic potential to describe the system is the Gibbs energy $G$. At constant external pressure $P=P^{\alpha}$ and temperature $T$, the variation of the Gibbs energy is given by

$$
d G=d U-T d S+P^{\alpha} d V,
$$

where $U$ is the internal energy and $S$ is the entropy. The change in internal energy in the $\alpha$ phase is

$$
d U^{\alpha}=T d S^{\alpha}-P^{\alpha} d V^{\alpha}+\sum_{i=1}^{k} \mu_{i}^{\alpha} d N_{i}^{\alpha},
$$

where $\mu_{i}^{\alpha}$ is the chemical potential of species $i$ and $V_{\alpha}$ is the volume of the $\alpha$ phase. We assign the surface energy to the $\beta$ phase [29], thus

$$
d U^{\beta}=T d S^{\beta}-P^{\beta} d V^{\beta}+\sum_{i=1}^{k} \mu_{i}^{\beta} d N_{i}^{\beta}+\sigma d A^{\beta},
$$

where $A^{\beta}$ is the surface of the spherical nucleus and $V_{\beta}$ is the volume of the $\beta$ phase. The variation of the total Gibbs energy of the system is given by [37]

$$
d G=-\left(P^{\beta}-P^{\alpha}-\frac{2 \sigma}{R_{\beta}}\right) d V^{\beta}+\sum_{i=1}^{k}\left(\mu_{i}^{\beta}-\mu_{i}^{\alpha}\right) d N_{i}^{\beta} .
$$

The condition of thermodynamic equilibrium, $d G=0$, leads to the equality of the chemical potentials,

$$
\mu_{i}^{\alpha, *}=\mu_{i}^{\beta, *} \quad(i=1 \ldots k)
$$

and to the Laplace relation,

$$
P^{\beta, *}-P^{\alpha}=\frac{2 \sigma}{R_{\beta}^{*}},
$$

where the stars represent equilibrium $(d G=0)$. Equations (6) and (7) are the classical equilibrium conditions. The GibbsDuhem equation for the $\beta$ phase to which the surface is assigned is

$$
S^{\beta} d T-V^{\beta} d P^{\beta}+\sum_{i=1}^{k} N_{i}^{\beta} d \mu_{i}^{\beta}+A^{\beta} d \sigma=0 .
$$

We assume that the surface tension does not depend on composition. For an incompressible $\beta$ phase, the integration of the Gibbs-Duhem relation leads to the following expression of the chemical potential:

$$
\mu_{i}^{\beta}\left(P^{\beta}\right)=\mu_{i}^{\beta}\left(P^{\alpha}\right)+v_{i}^{\beta}\left(P^{\beta}-P^{\alpha}\right),
$$

where we have used

$$
\left.\frac{\partial \mu_{i}^{\beta}}{\partial P^{\beta}}\right|_{T, N_{i}^{\beta}}=v_{i}^{\beta}
$$

with $v_{i}^{\beta}$ the atomic volume of species $i$ in $\beta$. Using the equilibrium conditions required by Eqs. (6) and (7) yields

$$
\Delta \mu_{i}^{*}=\frac{2 \sigma v_{i}^{\beta}}{R_{\beta}^{*}}
$$

where $\Delta \mu_{i}{ }^{*}$ is the difference in chemical potential between the $\alpha$ and $\beta$ phases at equilibrium and at the pressure $P^{\alpha}$. Equation (11) is the generalization of the Gibbs-Thomson equation for multicomponent systems.

\section{A. Infinite domain}

We first consider the $\alpha$ phase as infinite. In this case, the formation of the critical nucleus has no effect on the composition in $\alpha$ that remains equal to the initial composition, denoted $c_{i}^{\alpha, 0}$. The Gibbs-Thomson relation for the critical nucleus writes

$$
\Delta \mu_{i}^{*}=\mu_{i}^{\alpha, 0}-\mu_{i}^{\beta}\left(c_{2}^{\beta, *}, c_{3}^{\beta, *}, \ldots, c_{k}^{\beta, *} P^{\alpha}\right)=\frac{2 \sigma v_{i}^{\beta}}{R_{\beta}^{*}}
$$

with $\mu_{i}^{\alpha, 0}=\mu_{i}^{\alpha}\left(c_{2}^{\alpha, 0}, c_{3}^{\alpha, 0}, \ldots, c_{N}^{\alpha, 0}, P^{\alpha}\right)$ the chemical potential of species $i$ when the $\alpha$ phase contains no nucleus, i.e., $c_{i}^{\alpha}=c_{i}^{\alpha, 0}$ and $c_{i}^{\beta, *}$ is the atomic fraction of species $i$ in the critical nucleus. In an infinite matrix, the stationary solution is unique and corresponds to a saddle point of the change in Gibbs energy, $\Delta G\left(R_{\beta}, c_{i}^{\beta}\right)$, associated to the formation of a nucleus with radius $R_{\beta}$ at the composition $c_{i}^{\beta}$. Thus, the Gibbs-Thomson relations [Eq. (12)] form a system of $k$ equations with $k$ unknowns $(k-1$ independent nucleus 
compositions and the nucleus size) and completely define the composition and size of the critical nucleus. Rearranging Eq. (12) leads to

$$
\Delta \mu_{i}^{*}=\frac{v_{i}^{\beta}}{v_{1}^{\beta}} \Delta \mu_{1}^{*}, \quad i=2 \ldots k
$$

that gives the rule of parallel tangent (in binary) for no difference in atomic volume. Equation (13) sets the composition of the critical nucleus, which is independent of the surface tension; this is not the case for the critical radius. Another way of determining the properties of the critical nucleus is to solve the usual relations

$$
\frac{\partial \Delta G}{\partial R_{\beta}}=0, \quad \frac{\partial \Delta G}{\partial c_{i}^{\beta}}=0 .
$$

The work of formation in the NPT ensemble is given by

$$
\begin{aligned}
\Delta G & =G\left(R_{\beta}, N_{i}^{\beta}\right)-G(0,0) \\
& =-\left(P^{\beta}-P^{\alpha}-\frac{3 \sigma}{R_{\beta}}\right) V^{\beta}+\sum_{i=1}^{k} N_{i}^{\beta}\left(\mu_{i}^{\beta}-\mu_{i}^{\alpha}\right) .
\end{aligned}
$$

Using Eq. (9) in Eq. (15) leads to the following classical expression for the work of formation:

$$
\Delta G=-\frac{4 \pi R_{\beta}^{3}}{3 v^{\beta}} \sum_{i=1}^{k} c_{i}^{\beta} \Delta \mu_{i}+4 \pi R_{\beta}^{2} \sigma,
$$

where $v^{\beta}=\sum_{i=1}^{k} c_{i}^{\beta} v_{i}^{\beta}$ and where we recognize the driving force for nucleation $\sum_{i=1}^{k} c_{i}^{\beta} \Delta \mu_{i}$ with $\Delta \mu_{i}$ the difference in chemical potentials at the matrix pressure. In principle the work of formation depends on the nucleus composition [41-47]. The problem is simpler when nucleation can be reduced to one dimension; the process is then investigated in the size space only, $\{R\}$. In this case, the cluster composition is in equilibrium during nucleation for either thermodynamic or kinetic reasons as any deviations in composition instantaneously relax toward equilibrium. Such reduction to one dimension is possible when, for instance in a binary mixture, the Hessian of the Gibbs energy of the $\beta$ phase is very large [43]. In a more general case the properties of the clusters are set by the thermodynamic path defined by the steepest descent of the work of formation or any deviations from the minimum energy path due to kinetic effects, such as the presence of a low mobility component [42]. The work of formation of the critical nucleus is obtained by introducing the stationary solutions in Eq. (15),

$$
\Delta G^{*}=\frac{4 \pi \sigma R_{\beta}^{* 2}}{3}
$$

\section{B. Finite-size effects}

So far, the above thermodynamic description of classical nucleation in multicomponent systems does not include depletion effects. In a confined system, as the one depicted in Fig. 1, the formation of the nucleus affects the matrix compositions $c_{i}^{\alpha}$, which are set by the $k-1$ independent mass conservation equations, $N_{i}=N_{i}^{\alpha}+N_{i}^{\beta}$. In the following we assume that both phases have the same atomic volumes

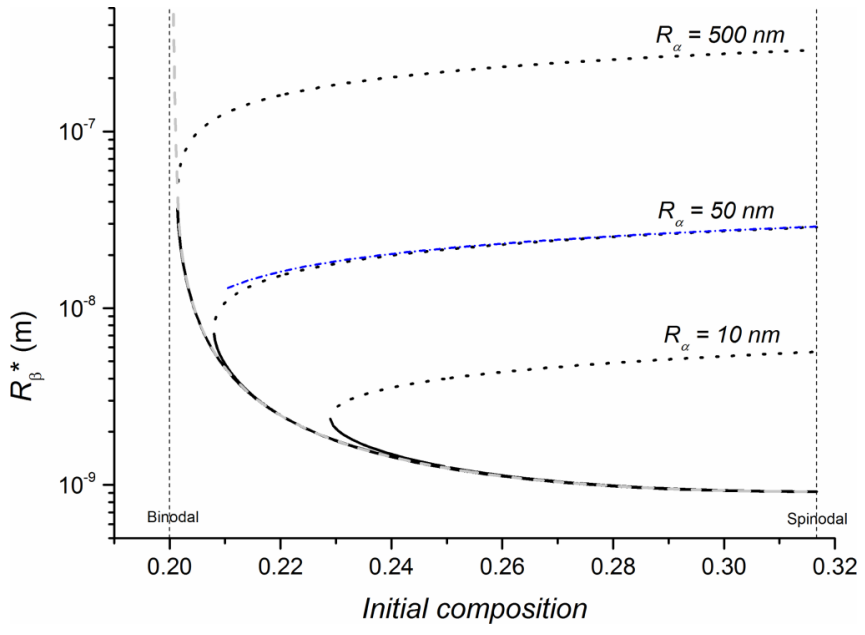

FIG. 2. Critical sizes of the nucleus in an infinite system (gray dashed line) and in finite systems for various system sizes (10, 50, and $500 \mathrm{~nm}$ ), as a function of the initial composition in $\alpha$. For $R_{\alpha}=10 \mathrm{~nm}$, nucleation is prohibited when the initial composition is below $\sim 0.228$, the lower part of the curve corresponds to the unstable solution $\left(R_{\beta}^{*}=R_{\beta}^{S P}\right.$ ), and the upper part to the stable solution $\left(R_{\beta}^{*}=R_{\beta}^{S S}\right)$. The blue dashed line corresponds to the approximate solution for the stable cluster [Eq. (28)] when $R_{\alpha}=50 \mathrm{~nm}$, strictly valid when $R_{\beta}^{S S} \gg R_{\beta}^{0}$.

(denoted $v$ ), which are composition independent, so as to conserve the total volume of the system described in Fig. 1 whatever the size and composition of the nucleus. Such an assumption can be justified, for example, in the context of solid state precipitation, as elastic effects that originate from atomic volume differences are known to come into play, if they are present, when clusters reach larger sizes, as compared with the critical radius, and are thus often neglected during the nucleation regime (as in Ref. [42]). In this case, the mass conservation gives

$$
c_{i}^{\alpha, 0} R_{\alpha}^{3}=c_{i}^{\beta} R_{\beta}^{3}+c_{i}^{\alpha}\left(R_{\alpha}^{3}-R_{\beta}^{3}\right) .
$$

This supplementary set of equations, combined with Eqs. (6) and (7), or with Eq. (11), defines the stationary solutions for confined multicomponent solutions. Note that the chemical potentials of the matrix phase are no longer equal to their initial values since the composition in $\alpha$ has changed. Another difference is that there are in general two stationary solutions for $R_{\beta}^{*}$, one corresponding to a saddle point of the work of formation, denoted $R_{\beta}^{S P}$, whereas the second solution corresponds to a stable state, denoted $R_{\beta}^{S S}$. This is depicted in Fig. 2, where the stationary states [solutions of Eqs. (6), (7), and (18)] are shown for various system sizes as a function of the initial composition. For this illustration in a binary system, we have used the regular solution model at a given value of $T / T_{c}(=0.8656)$, with $T_{c}$ the critical temperature, and for a given value of surface tension $\left(0.025 \mathrm{~J} / \mathrm{m}^{2}\right)$. As already mentioned, for a given supersaturation, nucleation is prohibited when the system is too small. For the example of Fig. 2, in the case $R_{\alpha}=10 \mathrm{~nm}$, nucleation is prohibited when the initial composition $c_{2}^{\alpha, 0}$ is below $\sim 0.228$. 

by

The work of formation in a confined system is now given

$$
\begin{aligned}
\Delta G= & -\left(P^{\beta}-P^{\alpha}-\frac{3 \sigma}{R_{\beta}}\right) V^{\beta}+\sum_{i=1}^{k} N_{i}^{\beta}\left(\mu_{i}^{\beta}-\mu_{i}^{\alpha}\right) \\
& +\sum_{i=1}^{k} N_{i}\left(\mu_{i}^{\alpha}-\mu_{i}^{\alpha, 0}\right)
\end{aligned}
$$

and the nucleation barrier by

$$
\Delta G^{*}=\frac{4 \pi \sigma R_{\beta}^{* 2}}{3}+\frac{4 \pi R_{\alpha}^{3}}{3 v} \sum_{i=1}^{k} c_{i}^{\alpha, 0}\left(\mu_{i}^{\alpha, *}-\mu_{i}^{\alpha, 0}\right),
$$

where $\mu_{i}^{\alpha, *}$ is the chemical potential in $\alpha$ at the stationary matrix composition $c_{i}^{\alpha, *}$. These expressions coincide with the results of Wilhelmsen and Reguera [37] in the NVT ensemble when the matrix pressure is fixed. The last term in Eq. (20) vanishes for infinite systems and the classical expression is recovered [Eq. (17)].

In general, the equilibrium conditions [Eqs. (6) and (7)] combined with the mass conservation equation [Eq. (18)] can only be solved numerically (as done for the example of Fig. 2).

In the limit of small supersaturation, the radius of the critical cluster can be written as $[42,48-50]$

$$
R_{\beta}^{*}=\frac{2 \sigma v}{\sum_{i=2}^{k} \sum_{j=2}^{k}\left(c_{i}^{\beta}-\bar{c}_{i}^{\alpha}\right) G_{i j}^{\alpha}\left(c_{j}^{\alpha, *}-\bar{c}_{j}^{\alpha}\right)}
$$

or in dyadic notation,

$$
R_{\beta}^{*}=\frac{2 \sigma v}{(\Delta \overline{\mathbf{c}})^{T} \mathrm{G}^{\alpha} \Delta \mathbf{c}^{\alpha, *}},
$$

where $\mathrm{G}^{\alpha}$ is the Hessian of the Gibbs energy of $\alpha$ evaluated at equilibrium composition $\bar{c}_{i}^{\alpha}, \Delta \overline{\mathbf{c}}=\bar{c}_{i}^{\beta}-\bar{c}_{i}^{\alpha}$ for $i=2 \ldots k$ is the equilibrium tie-line vector, and $\Delta \mathbf{c}^{\alpha, *}=c_{j}^{\alpha, *}-\bar{c}_{j}^{\alpha}$ for $j=2 \ldots k$ is the vector supersaturation. Here we introduce the vector $\Delta \mathbf{c}^{\alpha, 0}=c_{j}^{\alpha, 0}-\bar{c}_{j}^{\alpha}$ that represents the initial supersaturation, $c_{i}^{\alpha, *}=c_{i}^{\alpha, 0}$. Therefore, any cluster in equilibrium with the matrix at a given composition $\mathbf{c}^{\alpha}$ satisfies

$$
\frac{2 \sigma v}{R_{\beta}^{*}}=(\Delta \overline{\mathbf{c}})^{T} \mathrm{G}^{\alpha} \Delta \mathbf{c}_{\mathrm{dep}}^{*}+\frac{2 \sigma v}{R_{\beta}^{0}},
$$

where $\Delta \mathbf{c}_{\mathrm{dep}}^{*}=c_{j}^{\alpha, *}-c_{j}^{\alpha, 0}$ accounts for the depletion effects and where we have introduced the critical radius for an infinite system (in the low supersaturation limit) that is given by

$$
R_{\beta}^{0}=\frac{2 \sigma v}{(\Delta \overline{\mathbf{c}})^{T} \mathrm{G}^{\alpha} \Delta \mathbf{c}^{\alpha, 0}} .
$$

Thus, the two solutions $R_{\beta}^{S P}$ (the unstable one) and $R_{\beta}^{S S}$ (the stable one) obey Eq. (23) but for different vectors $\Delta \mathbf{c}_{\mathrm{dep}}^{*}=\Delta \mathbf{c}_{\mathrm{dep}}^{S P}$ and $\Delta \mathbf{c}_{\mathrm{dep}}^{*}=\Delta \mathbf{c}_{\mathrm{dep}}^{S S}$. We then introduce the low supersaturation approximation in the mass conservation equation. We first transform Eq. (18)

$$
\Delta \mathbf{c}_{\mathrm{dep}}=-\Delta \overline{\mathbf{c}}\left(\frac{R_{\beta}}{R_{\alpha}}\right)^{3}
$$

with $\Delta \mathbf{c}_{\mathrm{dep}}=c_{j}^{\alpha}-c_{j}^{\alpha, 0}$ and where we have used that $\overline{\mathbf{c}}^{\beta}-$ $\mathbf{c}_{\alpha}^{0} \approx \Delta \overline{\mathbf{c}}$ and both $\Delta \mathbf{c}^{\alpha} \ll \Delta \overline{\mathbf{c}}$ and $\Delta \mathbf{c}^{\beta} \ll \Delta \overline{\mathbf{c}}$ with $\Delta \mathbf{c}^{\beta}=$ $c_{j}^{\beta}-\bar{c}_{j}^{\beta}$ represents the deviation of the nucleus composition from equilibrium, which can be related to supersaturation as follows $[42,43]$ :

$$
\Delta \mathbf{c}^{\beta}=\left(\mathrm{G}^{\beta}\right)^{-1} \mathrm{G}^{\alpha} \Delta \mathbf{c}^{\alpha},
$$

where $\mathrm{G}^{\beta}$ is the Hessian of the Gibbs energy of $\beta$ evaluated at equilibrium composition $\bar{c}_{i}^{\beta}$. The deviation is therefore order of supersaturation and the condition $\Delta \mathbf{c}^{\beta} \ll \Delta \overline{\mathbf{c}}$ is guaranteed. Moreover, the derivation of Eq. (25) also requires $\left(R_{\beta} / R_{\alpha}\right)^{3} \ll$ 1 , which is a very good approximation provided $R_{\beta}$ and $R_{\alpha}$ differ by at least one order of magnitude. Finally, for both $R_{\beta}^{*}=R_{\beta}^{S P}$ and $R_{\beta}^{*}=R_{\beta}^{S S}$ (the two solutions in confined space) the equilibrium conditions together with the mass conservation can be written as

$$
2 \sigma v\left(\frac{1}{R_{\beta}^{*}}-\frac{1}{R_{\beta}^{0}}\right)=-(\Delta \overline{\mathbf{c}})^{T} \mathrm{G}^{\alpha} \Delta \overline{\mathbf{c}}\left(\frac{R_{\beta}^{*}}{R_{\alpha}}\right)^{3} .
$$

The stationary solutions of the nucleation problem in a confined system obey Eq. (27) in the limit of low supersaturation. Equation (27) is the main result of the present analysis as it relates the stationary solutions to the system size. For $R_{\alpha} \rightarrow+\infty$, Eq. (27) admits only one solution and the critical cluster size in infinite system is recovered, $R_{\beta}^{*}=R_{\beta}^{0}$. Equation (27) presents another interesting limit: when the system size is large enough, the stable solution is known to be much larger than the unstable solution (which is in this case very close to $R_{\beta}^{0}$ ) and thus

$$
R_{\beta}^{S S}=\left(\frac{2 \sigma v}{(\Delta \overline{\mathbf{c}})^{T} \mathrm{G}^{\alpha} \Delta \overline{\mathbf{c}} R_{\beta}^{0}}\right)^{1 / 3} R_{\alpha} .
$$

This approximate solution [Eq. (28)] compares well with the exact result, as shown in Fig. 2 , in the limit $R_{\beta}^{S S} \gg R_{\beta}^{0}$. Equation (28) allows for the prediction of the final state with a very good accuracy provided $R_{\beta}^{S S} \gg R_{\beta}^{0}$. We are interested in

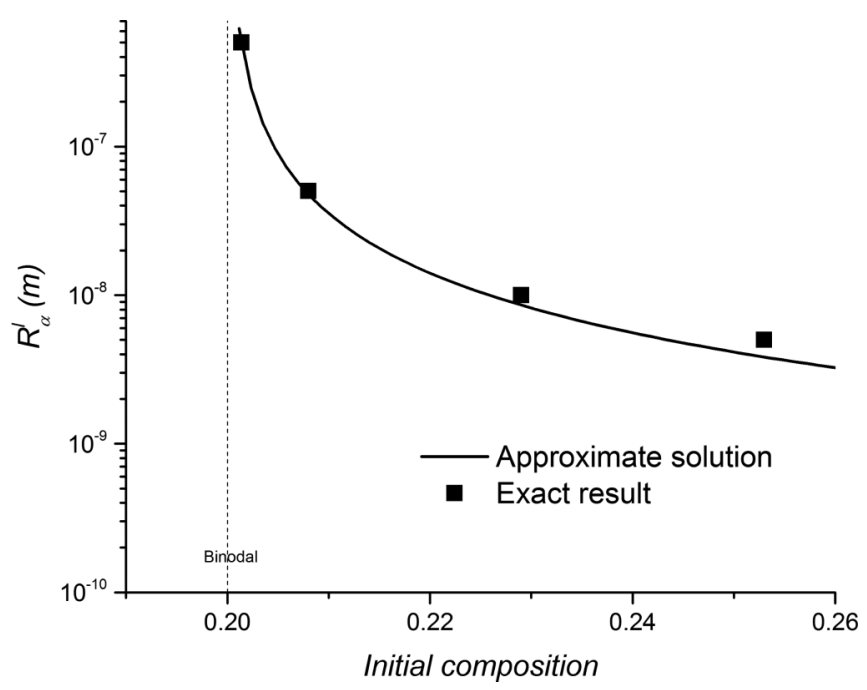

FIG. 3. System size below which nucleation is prohibited as a function of the initial composition. 
the superstabilization effect that takes place when Eq. (27) has no solution rather than the previously mentioned two solutions (see Ref. [51] for more details on quartic equations). Such a criterion gives the system size, $R_{\alpha}^{l}$, below which nucleation is prohibited,

$$
R_{\alpha}^{l}=\left(a \frac{(\Delta \overline{\mathbf{c}})^{T} \mathrm{G}^{\alpha} \Delta \overline{\mathbf{c}}}{2 \sigma v}\right)^{1 / 3}\left(R_{\beta}^{0}\right)^{4 / 3}
$$

with $a=256 / 27$, a constant. For $R_{\alpha}<R_{\alpha}^{l}$ nucleation is impossible as the initial supersaturation is not large enough; combined with Eq. (24), the above condition also provides the minimum supersaturation for a given system size $R_{\alpha}$. This criterion on size is compared with the exact result in Fig. 3 and a very good agreement is found even for a relatively high initial composition, provided the approximation $\left(R_{\beta}^{*} / R_{\alpha}^{l}\right)^{3} \ll 1$ remains valid.

\section{CONCLUSION}

In this work we have derived an approximate solution for the container size below which nucleation is prohibited; this allows for a very good prediction of the superstabilization effect, one of the most interesting peculiarities of nucleation in confined spaces. From our simple result, the prediction of the superstabilization effect requires the knowledge of only a few thermodynamic parameters: the equilibrium tie line, the Hessian of the Gibbs energy density of the mother phase evaluated at equilibrium, and the surface energy. Thus, with the widespread availability of thermodynamic databases, the present analysis could be used in the design of novel nanomaterials.

\section{ACKNOWLEDGMENT}

We are grateful for the financial support of the French ANR Agency (DIAMONS project).
[1] J. W. Gibbs, Trans. Connecticut Acad. Sci. 3, 343 (1875); see also The Collected Works (Longmans Green, New York, 1928), Vol. 1.

[2] J. D. van der Waals and Ph. Kohnstamm, Lehrbuch der Thermodynamik (Johann- Ambrosius-Barth, Leipzig, 1908).

[3] J. S. Rowlinson (translation of J. D. van der Waals), J. Stat. Phys. 20, 197 (1979); German version: J. D. Van der Waals, Z. Phys. Chem. 13, 657 (1893).

[4] M. Volmer, Kinetik der Phasenbildung (Steinkopff, Dresden/Leipzig, 1939).

[5] R. Becker and W. Doring, Ann. Phys. (Leipzig) 416, 719 (1935).

[6] J. Zeldovich, J. Exp. Theor. Phys. (USSR) 12, 525 (1942).

[7] Y. I. Frenkel, Kinetic Theory of Liquids (Oxford University Press, Oxford, 1946).

[8] Nucleation, edited by A. C. Zettlemoyer (Marcel Dekker, New York, 1969).

[9] D. Turnbull and J. C. Fisher, J. Chem. Phys. 17, 71 (1949).

[10] J. Feder, K. C. Russell, J. Lothe, and G. M. Pound, Adv. Phys. 15, 111 (1966).

[11] K. C. Russell, Adv. Colloid Interface Sci. 13, 205 (1980).

[12] H. Reiss, J. Chem. Phys. 18, 840 (1950).

[13] D. Stauffer, J. Aerosol Sci. 7, 319 (1976).

[14] G. Wilemski, J. Chem. Phys. 110, 6451 (1999).

[15] H. Trinkaus, Phys. Rev. B 27, 7372 (1983).

[16] F. F. Abraham, Homogeneous Nucleation Theory (Academic, New York, 1974).

[17] H. Vehkamaki, Classical Nucleation Theory in Multicomponent Systems (Springer, New York, 2006).

[18] K. F. Kelton and A. L. Greer, Nucleation in Condensed Matter: Applications in Materials and Biology (Pergamon, New York, 2010).

[19] R. S. Berry, Nature (London) 393, 212 (1998).

[20] M. Schmidt, R. Kusche, B. von Issendorff, and H. Haberland, Nature (London) 393, 238 (1998).

[21] M. Schmidt, R. Kusche, T. Hippler, J. Donges, W. Kronmuller, B. von Issendorff, and H. Haberland, Phys. Rev. Lett. 86, 1191 (2001).
[22] A. P. Alivisatos, Science 271, 933 (1996).

[23] C. P. Collier, R. J. Saykally, J. J. Shiang, S. E. Henrichs, and J. R. Heath, Science 277, 1978 (1997).

[24] V. Dubroskii, Nucleation Theory and Growth of Nanostructures (Springer, New York, 2014).

[25] A. Vorobev, D. Lyubimov, and T. Lyubimova, Phys. Rev. E 95, 022803 (2017)

[26] M. Rao, B. J. Berne, and M. H. Kalos, J. Chem. Phys. 68, 1325 (1978).

[27] V. Talanquer and D. W. Oxtoby, J. Chem. Phys. 100, 5190 (1994).

[28] Z. Kozisek, P. Demo, and A. M. Sveshnikov, J. Chem. Phys. 125, 114504 (2006).

[29] Z. Kozisek, K. Sato, S. Ueno, and P. Demo, J. Chem. Phys. 134, 094508 (2011).

[30] Z. Kozisek and P. Demo, J. Chem. Phys. 126, 184510 (2007).

[31] F. Schweitzer and L. Schimansky-Geier, J. Colloid Interface Sci. 119, 67 (1987).

[32] A. S. Abyzov and J. W. P. Schmelzer, J. Chem. Phys. 127, 114504 (2007).

[33] J. W. P. Schmelzer and A. S. Abyzov, J. Chem. Phys. 134, 054511 (2011)

[34] D. Reguera, R. K. Bowles, Y. Djikaev, and H. Reiss, J. Chem. Phys. 118, 340 (2003).

[35] Ø. Wilhelmsen, D. Bedeaux, and S. Kjelstrup, and D. Reguera, J. Chem. Phys. 141, 071103 (2014).

[36] Ø. Wilhelmsen, D. Bedeaux, S. Kjelstrup, and D. Reguera, J. Chem. Phys. 140, 024704 (2014).

[37] Ø. Wilhelmsen and D. Reguera, J. Chem. Phys. 142, 064703 (2015).

[38] J. Schmelzer and H. Ulbricht, J. Colloid Interface Sci. 117, 325 (1987).

[39] M. A. Duran-Olivencia and J. F. Lutsko, Phys. Rev. E91, 022402 (2015).

[40] J. F. Lutsko, J. Chem. Phys. 137, 154903 (2012).

[41] D. Reguera and H. Reiss, J. Chem. Phys. 119, 1533 (2003).

[42] T. Philippe, D. Blavette, and P. W. Voorhees, J. Chem. Phys. 141, 124306 (2014). 
[43] T. Philippe, M. Bonvalet, and D. Blavette, J. Chem. Phys. 144, 204501 (2016).

[44] T. Philippe, J. Chem. Phys. 142, 094501 (2015).

[45] T. Philippe and D. Blavette, Philos. Mag. 91, 4606 (2011).

[46] T. Philippe and D. Blavette, Scr. Mater. 67, 77 (2012).

[47] T. Philippe and D. Blavette, J. Chem. Phys. 135, 134508 (2011).
[48] A. Umantsev and G. B. Olson, Scr. Metall. Mater. 29, 1135 (1993).

[49] J. E. Morral and G. R. Purdy, Scr. Metall. Mater. 30, 905 (1994). [50] J. J. Hoyt, Acta Mater. 47, 345 (1999).

[51] D. E. Smith, A Source Book in Mathematics (Dover, New York, 1994). 